\title{
C/EBPbeta mediates bortezomib-induced EBV and KSHV lytic gene expression
}

\author{
Courtney O'Farrell, Meir Shamay, Nene Kalu, Richard F Ambinder \\ From $12^{\text {th }}$ International Conference on Malignancies in AIDS and Other Acquired Immunodeficiencies \\ (ICMAOI) \\ Bethesda, MD, USA. 26-27 April, 2010
}

Bortezomib is a proteasome inhibitor used clinically for the treatment of multiple myeloma and mantle cell lymphoma and is being evaluated for the treatment of AIDS-related lymphoma and Kaposi's sarcoma in the AIDS Malignancy Consortium. Activation of herpes zoster infection is a well-recognized complication of bortezomib therapy. In previous work, we have shown that bortezomib is a potent inducer of EBV and KSHV lytic gene expression and demonstrated a novel approach to therapy that leverages this activation to facilitate tumor imaging and treatment.

In an effort to better understand the relevant pathways, we confirmed that bortezomib at $20 \mathrm{nM}$ concentrations induces expression of EBV and KSHV immediate early genes (EBV ZTA, KSHV RTA) as assessed by immunoblot and by reverse transciptase PCR for the associated transcripts. Bortezomib activates EBV ZTA promoter expression and KSHV RTA promoter expression in reporter assays. C/EBPalpha and beta protein family members are recognized activators of EBV ZTA and KSHV RTA expression. Bortezomib does not alter C/EBPalpha protein level as assessed by immunoblot but does lead to increased C/EBPbeta. C/EBPbeta includes activating isoforms (LAP) and an inhibitory isoform (LIP). In reporter assays, we show that C/EBPbeta LAP activates the EBV promoters whereas LIP is inhibitory. In ChIP assays we show that bortezomib treatment leads to increased C/EBPbeta binding to the ZTA promoter. Mutation of the binding sites abolishes activation of the promoter associated with bortezomib treatment. In order to determine whether C/EBPbeta expression simply tracks with lytic viral gene expression or plays a key role in mediating such expression, we created a tetregulated $\mathrm{C} / \mathrm{EBPb}$ ta knockdown. In the presence of doxycycline, C/EBPbeta is suppressed, and bortezomib lytic induction of EBV as measured by increases in ZTA mRNA, ZTA protein, and viral DNA copy number are all blunted. Induction of lytic viral gene expression in EBV Akata cell lines by anti-Ig treatment is also blunted.

These results suggest that C/EBPbeta family members play a key role in mediating activation of EBV and KSHV lytic infection following bortezomib and other inducers. $^{1}$

\section{Acknowledgements}

This work is supported by P30CA06973 and P50CA96888.

This article has been published as part of Infectious Agents and Cancer Volume 5 Supplement 1, 2010: Proceedings of the $12^{\text {th }}$ International Conference on Malignancies in AIDS and Other Acquired

Immunodeficiencies (ICMAOI). The full contents of the supplement are available online at http://www.biomedcentral.com/1750-9378/5?issue=S1.

Published: 11 October 2010

Reference

1. Fu DX, et al: Bortezomib-induced enzyme-targeted radiation therapy in herpesvirus-associated tumors. Nat Med 2008, 14:1118-1122.

\section{doi:10.1186/1750-9378-5-S1-A8}

Cite this article as: O'Farrell et al:: C/EBPbeta mediates bortezomibinduced EBV and KSHV lytic gene expression. Infectious Agents and Cancer 2010 5(Suppl 1):A8.

\footnotetext{
*Correspondence: cofarre1@jhmi.edu

Department of Oncology, Johns Hopkins School of Medicine, Baltimore, MD, USA

Full list of author information is available at the end of the article
} 